- Nota -

\title{
LAS NEGOCIACIONES INTERNACIONALES SOBRE EL CAMBIO CLIMÁTICO: RUMBO A LA COP16
}

\author{
Paola MilenKa Villavicencio Calzadilla* \\ Becaria predoctoral \\ Universitat Rovira i Virgili \\ p_villavicencio@hotmail.com
}

Recibido: 19 de octubre de 2010 / Aceptado: 18 de noviembre de 2010

\section{RESUMEN:}

Después del fracaso de la XV Conferencia de Naciones Unidas sobre el Cambio Climático celebrada en la ciudad de Copenhague, Dinamarca, y tras el debate de diversas iniciativas populares de solución a la crisis climática en la Primera Conferencia Mundial de los Pueblos sobre el Cambio Climático, desarrollada en la ciudad de Cochabamba, Bolivia, aún se mantiene la última esperanza para que en Cancún se obtenga un acuerdo jurídicamente vinculante que fortalezca el régimen jurídico del cambio climático antes de que el Protocolo de Kioto llegue a su fin en el 2012. No obstante, las probabilidades de un nuevo fracaso parecen ser cada día más evidentes.

\section{RESUM:}

Després del fracàs de la XV Conferència de Nacions Unides sobre el canvi climàtic (COP15) celebrada a la ciutat de Copenhaguen, Dinamarca, i després del debat de diverses iniciatives populars de solució a la crisi climàtica durant la Primera Conferència Mundial dels Pobles sobre el Canvi Climàtic, desenvolupada a la ciutat de Cochabamba, Bolívia, encara es manté l'última esperança perquè a Cancun s'obtingui

\footnotetext{
*** Becaria predoctoral del Ministerio de Asuntos Exteriores y de Cooperación de España y la Agencia Española de Cooperación Internacional para el Desarrollo (MAEC-AECID), adscrita al Departamento de Derecho Público de la Universidad Rovira i Virgili (URV) y al Centro de Estudios de Derecho Ambiental de Tarragona (CEDAT).
} 
un acord jurídicament vinculant que enforteixi el règim jurídic del canvi climàtic després que el Protocol de Kyoto s'acabi el 2012. No obstant això, les probabilitats d'un nou fracàs semblen ser cada dia més evidents.

\begin{abstract}
:
After the failure of the Fifteenth United Nations Conference on Climate Change (COP15) held in Copenhagen, Denmark, and the various popular solutions to the climate crisis that were debated during the first World People's Conference on Climate Change, held in the city of Cochabamba, Bolivia, there is still hope that at Cancun a legally binding agreement will be entered into that will strengthen the legislation governing climate change before the Kyoto Protocol comes to an end in 2012. However, the chances of another failure appear to be increasingly evident.
\end{abstract}

PALABRAS CLAVE: cambio climático — Protocolo de Kioto - Conferencia de las Partes (Cancún)

PARAULES CLAU: Canvi climàtic — Protocol de Kyoto - Conferència de les Parts (Cancún)

KEYWORDS: Climate change - Kyoto Protocol - Conference of the Parties (Cancun)

\footnotetext{
Sumario: I. Introducción. La lucha internacional contra el cambio climático. II. Las negociaciones intergubernamentales: el Acuerdo de Copenhague. III. Las negociaciones no gubernamentales: la Conferencia de los Pueblos sobre el Cambio Climático. IV. Desde Bonn a Tianjin, ¿cuál es el escenario para Cancún? V. Consideraciones finales (el reto en Cancún: un acuerdo jurídicamente vinculante). VI. Bibliografía. 1. Referencias doctrinales. 2. Referencias documentales. 3. Referencias normativas.

\section{INTRODUCCIÓN. LA LUCHA INTERNACIONAL CONTRA EL CAMBIO CLIMÁTICO}


Los distintos episodios de sequías e inundaciones acaecidos durante el segundo semestre del 2010 permiten apreciar que el cambio climático está produciendo efectos devastadores en el planeta. Así por ejemplo, los incendios forestales registrados en Rusia, que afectaron a gran parte de la población, ${ }^{1}$ y las inundaciones en Pakistán y China, que provocaron la muerte de miles de personas durante las últimas semanas de septiembre del $2010^{2}$, son solo algunos ejemplos de los desastres naturales provocados por influencia directa del cambio climático. Si bien los países en desarrollo están sufriendo las consecuencias de los cambios de temperatura, los países desarrollados y principales causantes del calentamiento global también se ven altamente amenazados, situación que ha llamado la atención de la sociedad en general.

La preocupación por el calentamiento atmosférico y por los efectos que este fenómeno pueda provocar sobre el planeta no es reciente; por el contrario, esta inquietud data de hace varios años. A partir de la publicación de una serie de informes científicos elaborados por el Panel Intergubernamental sobre Cambio Climático (IPCC, por sus siglas en inglés $)^{3}$, el cambio climático empezó a ocupar un lugar importante en las discusiones de política internacional, pues los Estados y sus gobiernos se dieron cuenta de la gran amenaza que este representaba y que, por lo tanto, debían diseñar alternativas de actuación. En consecuencia, dado que el cambio climático es un problema mundial que puede afectar en mayor o menor medida a cualquier país, los Estados suscribieron el Convenio Marco de Naciones Unidas sobre el Cambio Climático (en adelante, CMNUCC) $)^{4}$ y el Protocolo de Kioto 5 , en 1992 y 1997, respectivamente.

\footnotetext{
${ }^{1}$ A finales de julio del 2010 se registró en Rusia la peor ola de calor en 130 años, que alcanzó los $40^{\circ} \mathrm{C}$ de temperatura y provocó hasta 843 incendios forestales, que afectaron a cientos de familias y causaron la muerte de 52 personas. Estos incendios afectaron a una superficie de 430.000 hectáreas. Vid. ASOCIACIÓN INTERNACIONAL DE GESTORES DE EMERGENCIA (IAEM-España), "Incendios Forestales en Rusia", actualizado el 8 de septiembre de 2010. Recuperado el 23 de septiembre de 2010, de: http://iaem.es/index.php?option=com_content\&view=article\&id=1529\&Itemid=155.

2 En los últimos meses, cerca de 1.500 personas fallecieron en China a causa de las inundaciones y más
de 90.000 fueron evacuadas a causa del desbordamiento del río Yalu. BBC MUNDO (2010, 22 de agosto)
"Corea del Norte y China afectadas por inundaciones". Recuperado el 20 de septiembre de 2010 , de:
http://www.bbc.co.uk/mundo/internacional/2010/08/100822_inundaciones_china_corea_norte_aw.shtml.
3 Los informes científicos emitidos por el Panel Intergubernamental sobre Cambio Climático pueden
consultarse en el portal oficial del IPCC: http://www.ipcc.ch/index.htm. Última visita, 20 de septiembre
de 2010 .
}

${ }^{4}$ BOE núm. 27, de 1 de febrero de 1994.

${ }^{5}$ BOE núm. 33, de 8 de febrero de 2005. 
Si bien la respuesta de política internacional al cambio climático comenzó con la adopción del CMNUCC, que se convirtió en el primer paso dentro del desarrollo del régimen jurídico que lo regula, la aprobación del Protocolo de Kioto en 1997 constituye un hito dentro de los esfuerzos globales. Con la finalidad de proteger el medio ambiente y alcanzar un desarrollo sostenible, el Protocolo es el primer instrumento con el que los países desarrollados y aquellos en transición hacia una economía de mercado ${ }^{6}$ - asumiendo la responsabilidad de sus actividades contaminantes - establecieron objetivos cuantitativos de límite o reducción de sus emisiones de gases de efecto invernadero (en adelante, GEI) ${ }^{7}$ para el período comprendido entre el año 2008 y el 2012 (el primer período de compromisos del Protocolo de Kioto) ${ }^{8}$.

Desde ese momento, la urgencia por lograr nuevos compromisos de reducción de emisiones de GEI y la necesidad de establecer medidas que permitan la adaptación al cambio climático han propiciado la celebración de reuniones de diversa índole; por una parte, se encuentran las conferencias de las partes del CMNUCC, celebradas en Bali en el $2007^{9} \mathrm{y}$ en Copenhague en el $2009^{10}$ como reuniones oficiales de los Estados; y, por otra, están las reuniones civiles, como la Conferencia de los Pueblos sobre el Cambio Climático y los

\footnotetext{
${ }^{6}$ Países conocidos dentro del CMNUCC como partes del anexo I.

7 El artículo 3.1 del Protocolo establece: "Las Partes incluidas en el anexo I se asegurarán, individual o conjuntamente, de que sus emisiones antropógenas agregadas, expresadas en dióxido de carbono equivalente, de los gases de efecto invernadero enumerados en el anexo A no excedan de las cantidades atribuidas a ellas, calculadas en función de los compromisos cuantificados de limitación y reducción de las emisiones consignados para ellas en el anexo B y de conformidad con lo dispuesto en el presente artículo, con miras a reducir el total de sus emisiones de esos gases a un nivel inferior en no menos de 5\% al de 1990 en el período de compromiso comprendido entre el año 2008 y el 2012”.

${ }^{8}$ Artículos 3.1 y 7 del Protocolo de Kioto.

9 El resultado de la XIII Conferencia de Naciones Unidas sobre el Cambio Climático (en adelante, COP13) y del tercer período de sesiones de la Conferencia de las Partes - que sirvió como reunión de las partes del Protocolo de Kioto- (en adelante, COP-RP3), desarrollada en Bali del 3 al 15 de diciembre de 2007, fue el Plan de Acción de Bali (Decisión 1/CP.13). En este documento se decidió crear un grupo de trabajo específico bajo el mandato de Naciones Unidas para el cambio climático que agrupe a todos los países firmantes del Convenio y que debe emprender negociaciones formales que den como resultado un documento que pueda sustituir al Protocolo de Kioto a partir del 2012. Vid. CONVENIO MARCO DE NACIONES UNIDAS SOBRE EL CAMBIO CLIMÁTICO, Decisión 1/CP.13 del Doc. FCCC/CP/ 2007/6/Add.1 de 14 de marzo de 2008, pp. 3-8. Recuperado el 20 de septiembre de 2010, de: http:// unfccc.int/resource/docs/2007/cop13/spa/06a01s.pdf\#page=3.

${ }^{10}$ La XV Conferencia de Naciones Unidas sobre el Cambio Climático y el quinto período de sesiones de la Conferencia de las Partes - que sirvió como reunión de las partes del Protocolo de Kioto- (COP15RP5), desarrollado en Copenhague del 7 al 18 de diciembre de 2009, tuvo como resultado el denominado Acuerdo de Copenhague. Vid. Decisión 2/CP.15 del Doc. FCCC/CP/2009/11/Add.1 de 30 de marzo de 2010, pp. 5-10. Recuperado el 20 de septiembre de 2010, de: http:/unfccc.int/resource/docs/2009/cop15/ spa/11a01s.pdf\#page $=4$.
} 
Derechos de la Madre Tierra celebrada en Bolivia en el 201011. Ambos ejemplos son una clara muestra de las iniciativas, diferentes pero compartidas, que la comunidad internacional ha desarrollado con la finalidad de lograr un acuerdo jurídicamente vinculante que dé continuidad al Protocolo de Kioto.

Estos escenarios de debate han generado dos discursos de contenidos diferenciados pero con finalidades compartidas: el discurso gubernamental y el discurso no gubernamental. En las reuniones gubernamentales se ha puesto en evidencia que los intereses económicos de los Estados ocupan un lugar importante dentro de los debates; sin embargo, en las reuniones no gubernamentales, donde participa la sociedad civil, las pasiones se han desbordado y han desembocado en propuestas ambiciosas difíciles de ser aceptadas por todos los Estados.

Pese a ello, la diferencia de opiniones en relación con aspectos como la existencia del cambio climático, la contribución al incremento de los GEI por parte de los países desarrollados y la magnitud de sus efectos presentes y futuros, deben concluir en compromisos comunes para los Estados donde se acepte que las actuaciones antropocéntricas, sobre todo en los países desarrollados, son la causa principal del calentamiento global, y, por lo tanto, se necesita la adopción de medidas reales que ayuden a combatir el cambio climático, aunque para ello se tengan que discutir y considerar las ambiciosas propuestas que surjan del seno mismo de la sociedad.

Los obstáculos presentados durante las últimas reuniones de los Estados en torno al cambio climático y el fracaso en las negociaciones de futuros compromisos reflejan la urgente necesidad de trabajar de manera conjunta y responsable. En este sentido, la esperanza ahora se encuentra en Cancún, México, sede de la XVI Conferencia de Naciones Unidas sobre el Cambio Climático (en adelante, COP16) ${ }^{12}$ y en el sexto

\footnotetext{
${ }^{11}$ La Conferencia Mundial de los Pueblos sobre el Cambio Climático y los Derechos de la Madre Tierra, celebrada en Bolivia del 20 al 22 de abril de 2010 como muestra de rechazo al documento firmado en Copenhague en diciembre del 2009, tenía como objetivo principal la elaboración de una serie de propuestas relativas a la lucha contra el cambio climático para presentarlas a las partes del CMNUCC para su debate en la XVI Conferencia de Naciones Unidas sobre el Cambio Climático, que se celebra en México. Véase Proyecto de Texto de Negociación presentado por el Estado Plurinacional de Bolivia al grupo de trabajo especial sobre la cooperación a largo plazo en el marco del Convenio Marco de Naciones Unidas sobre el Cambio Climático. Recuperado el 25 de septiembre de 2010, de: http:// unfccc.int/resource/docs/2010/awglca10/eng/misc02.pdf.

12 Portal oficial de la XVI Conferencia de las Partes del Convenio Marco de Naciones Unidas sobre el Cambio Climático: http://cc2010.mx/es/. Última visita, 28 de septiembre de 2010.
} 
período de sesiones de la Conferencia de las Partes — que sirve como reunión de las partes del Protocolo de Kioto- (en adelante, COP/RP6), donde las partes del CMNUCC, bajo observación de todos los sectores de la sociedad, tendrán la responsabilidad de suscribir un acuerdo que establezca compromisos vinculantes de reducción de emisiones de GEI como medida para luchar contra el cambio climático. No obstante, la complejidad y los desacuerdos suscitados durante las reuniones preparatorias para la COP16 hacen prever una nueva desilusión a nivel internacional, la cual podría desencadenar un "calentamiento social".

\section{LAS NEGOCIACIONES INTERGUBERNAMENTALES: EL ACUERDO DE COPENHAGUE-}

Entre los días 7 y 18 de diciembre de 2009 la comunidad internacional orientó su atención a una de las reuniones más importantes para el régimen jurídico del cambio climático: la XV Conferencia de las Naciones Unidas sobre el Cambio Climático (en adelante, COP15) $)^{13}$.

Si bien el objetivo de la Conferencia era "la conclusión de un acuerdo jurídicamente vinculante sobre el clima, válido en todo el mundo y que se aplique a partir de 2012"14, las reuniones llevadas a cabo en Copenhague dieron lugar la suscripción de un documento denominado "Acuerdo de Copenhague"15. Este documento, voluntario e impreciso y que se asemeja más a un pacto político $^{16}$, no introduce modificaciones al

${ }^{13}$ La COP13, desarrollada en Bali, Indonesia adoptó dos documentos importantes: el Plan de Acción de Bali y la Hoja de Ruta de Bali. En este último acuerdo (sobre un proceso de dos años) se estableció la COP15 de 2009 como plazo final para concluir las negociaciones de los nuevos compromisos de las partes y la cooperación a largo plazo.

14 Vid. KIESSLING, C., Crisis ambiental global: COP 15 ¿éxito o fracaso? Análisis y prospectivas futuras. Ed. FLACSO, Argentina, 2010, p. 4. Recuperado el 23 de septiembre de 2010, de: http:// www.flacso.org.ar/rrii/web/wp-content/uploads/2010/09/Kiessling-Crisis-ambiental-global-COP-15.pdf.

15 Vid. Decisión 2/CP.15, cit. Es importante señalar que dentro del sistema jurídico internacional el sustantivo "acuerdo" se utiliza generalmente como una denominación de instrumentos jurídicos internacionales que denotan la existencia de un tratado, tal como lo establece la Convención de Viena sobre el Derecho de los Tratados de 1969 (art. 1.1 definiciones). En el caso del llamado Acuerdo de Copenhague, al no haber sido aceptado por el conjunto de los miembros de la Convención, carece de las condiciones necesarias para ser un instrumento jurídicamente vinculante que tenga la condición de acuerdo internacional. Vid. ESTRADA, R., "Acuerdo de Copenhague: las negociaciones sobre el cambio climático después de la XV Conferencia”, en Revista del Centro de Economía Internacional, núm. 17, 2010, p. 114-115. Recuperado el 22 de septiembre de 2010, de: http://www.cei.gov.ar/revista/17/ parte5b.pdf.

16 Sobre la naturaleza jurídica del Acuerdo de Copenhague, véase SINDICO, F., "The Copenhagen Accord and the future of the international climate change regime", en Revista Catalana de Dret Ambiental, vol. I - núm. 1, 2010, pp. 1-24. Recuperado el 23 de agosto de 2010, de: http://www.rcda.cat. 
único instrumento vinculante del régimen del cambio climático: el Protocolo de Kioto; por el contrario, solo fue considerado como una insuficiente declaración de intenciones para frenar el calentamiento global.

Elaborado por voluntad de algunos líderes políticos ${ }^{17}$, este acuerdo no tuvo el apoyo necesario para que las Naciones Unidas lo consideraran un acuerdo oficial, por lo que se convirtió en un instrumento voluntario al que podían adherirse los países que así lo quisieran.

Dada la discrecionalidad de este pacto político, algunos países en desarrollo como Tuvalu, Sudán, Nicaragua, Venezuela, Cuba y Bolivia manifestaron su rechazo y desinterés en adherirse a su contenido. Los representantes de estos países se opusieron a la posibilidad de reemplazar el Protocolo de Kioto por un documento cuyos objetivos no son vinculantes y que además impone obligaciones de reducción de emisiones de GEI a aquellos países que menos han contribuido al problema, y que olvida que los países desarrollados, principales contaminantes, no han cumplido sus compromisos de transferencia de tecnología y colaboración financiera asumidos en Kioto.

Ni la actitud positiva del ex secretario ejecutivo del CMNUCC Yvo de Boer, quien había manifestado que el encuentro en Copenhague mostraba a la comunidad internacional el interés de los gobiernos por llevar a cabo acciones que eviten el cambio climático $^{18}$, ni la declaración del presidente de los Estados Unidos, Barack Obama, de haber alcanzado un "avance significativo" con la firma del Acuerdo ${ }^{19}$, ni la postura de algunos países con economías emergentes como China, India y México, quienes habían

17 El consenso final del Acuerdo involucró únicamente a los Estados Unidos, China, India, Brasil y Sudáfrica. Vid. ESTRADA, R., “Acuerdo de Copenhague: las negociaciones sobre el cambio climático después de la XV Conferencia”, op. cit., p. 110.

18 El ex secretario ejecutivo del CMNUCC Yvo de Boer señaló que durante la COP15 se fomentaron las negociaciones sobre temas de gran importancia relacionados con el cambio climático, como el mejoramiento de los mecanismos de flexibilidad del Protocolo de Kioto. También argumentó que en esta reunión se lograron importantes avances en materia de medidas de adaptación, un nuevo mecanismo tecnológico, un marco de fomento de la capacidad y una estructura para la financiación a los países en desarrollo, entre otros logros. Vid. CONVENIO MARCO DE NACIONES UNIDAS SOBRE EL CAMBIO CLIMÁTICO, "Discurso público de Yvo de Boer, secretario ejecutivo", Bruselas, 14 de abril de 2010. Recuperado el 27 de septiembre de 2010, de: http://unfccc.int/files/portal_espanol/press/ statements/application/pdf/100414_speech_brussels_spa.pdf.

19 Vid. El Mundo (2009, 19 de diciembre) “Un avance significativo, pero insuficiente”. Recuperado el 12 de septiembre de 2010, de: http://www.elmundo.es/elmundo/2009/12/18/ciencia/1261172856.html. 
manifestado su interés por adoptar compromisos de reducción de $\mathrm{GEI}^{20}$, evitaron que el encuentro de Copenhague fuera considerado un nuevo fracaso dentro de las negociaciones del régimen jurídico del cambio climático ${ }^{21}$.

Si bien el objetivo principal del Acuerdo de Copenhague es evitar un incremento de la temperatura del planeta por encima de $\operatorname{los} 2{ }^{\circ} \mathrm{C}$, este documento no fija con claridad los objetivos de reducción de emisiones para el año 2050, e incluso deja de lado las recomendaciones del IPCC por las que se exhorta a los países desarrollados a reducir sus emisiones de GEI para el 2020 entre un $25 \%$ y un 40\% respecto a las que tenían en $1990^{22}$.

La falta de claridad en la definición de los compromisos de reducción de emisiones, así como la imprecisión de las medidas y los plazos para alcanzarlos, provocó la desilusión de muchos países en desarrollo, movimientos ecologistas y organizaciones no gubernamentales, que consideraron el Acuerdo de Copenhague como un documento insuficiente frente a la magnitud del problema.

Aunque el Acuerdo de Copenhague contiene las propuestas de reducción de emisiones de cada uno de los países que se han adherido a su contenido ${ }^{23}$, determina el monto de dinero que los países desarrollados han de destinar a la mitigación y adaptación al cambio climático $^{24}$, y crea el Fondo Verde para el cambio climático y el mecanismo de transferencia de tecnología, no es más que una declaración política que evidencia el

\footnotetext{
${ }^{20}$ Cabe mencionar que, aunque países como China o Brasil han manifestado su voluntad de implicarse en la reducción de emisiones de GEI, la actuación de estos y otros países en desarrollo se ve condicionada por la colaboración financiera que puedan recibir y por un acuerdo vinculante en el que se establezcan medidas de reducción de los GEI a los países desarrollados. Vid. La Vanguardia (7 de octubre de 2010) "China condiciona la reducción de sus emisiones al dinero y la tecnología que reciba". Recuperado el 12 de octubre de 2010, de: http://www.lavanguardia.es/ciudadanos/noticias/20101007/54020261184/chinacondiciona-la-reduccion-de-sus-emisiones-al-dinero-y-la-tecnologia-que-reciba.html.

${ }^{21}$ Debido a que el texto final de la COP15 solo fue negociado en una reunión cerrada celebrada por algunos líderes políticos, lo que provocó que algunos países en desarrollo se sintieran excluidos y rechazaran su contenido, la COP15 concluyó sin consenso sobre el Acuerdo de Copenhague y por ello solo se "tomo nota" de su contenido. Vid. Acuerdo de Copenhague, cit. p. 4.

${ }^{22}$ Las recomendaciones del IPCC para responsables de políticas pueden verse en el Informe del Grupo de Trabajo III: Mitigación del IPCC, disponible en INTERGOVERNMENTAL PANEL ON CLIMATE CHANGE (IPCC), Third Assessment Report: Climate Change, 2001 (TAR). Recuperado el 9 de septiembre de 2010, de: http://www.grida.no/climate/ipcc_tar/vol4/spanish/pdf/wg3sum.pdf.

${ }^{23}$ Sobre las propuestas de reducción de emisiones realizadas por algunos países, véase SINDICO, F., "The Copenhagen Accord", cit., anexos II y III.

${ }^{24} 30.000$ millones de dólares entre el 2010 y el 2012, y de 100.000 millones de dólares anuales a partir del 2020.
} 
fracaso de los Estados, que no pudieron llegar a un mínimo consenso entre ellos para definir las estrategias de lucha y prevención contra el cambio climático ${ }^{25}$. Por consiguiente, la próxima Conferencia de las Partes, la COP16, será el escenario oportuno para que los Estados reivindiquen sus actuaciones y diseñen un acuerdo ambicioso, justo y vinculante que fortalezca el régimen jurídico internacional del cambio climático.

\section{LAS NEGOCIACIONES NO GUBERNAMENTALES: LA CONFERENCIA DE LOS PUEBLOS SOBRE EL CAMBIO CLIMÁTICO}

Durante el primer trimestre del 2010, las partes del CMNUCC que habían manifestado su acuerdo con las negociaciones llevadas a cabo en la COP15 asumieron la tarea de presentar sus compromisos voluntarios de reducción de emisiones según lo había establecido el propio Acuerdo de Copenhague ${ }^{26}$.

Paralelamente al desarrollo de este proceso posterior a Copenhague y bajo el liderazgo del presidente de Bolivia, Evo Morales, algunos países en desarrollo que manifestaron su desacuerdo con el documento suscrito en la ciudad danesa decidieron llevar a cabo una de las mayores reuniones sobre el cambio climático nunca antes realizada ${ }^{27}$, que pretendía dar voz a los movimientos civiles y a la sociedad en general: la Conferencia

\footnotetext{
${ }^{25}$ Vid. URRUTIA, O., "El régimen jurídico internacional del cambio climático después del 'Acuerdo de Copenhague'", en Revista de Derecho de la Pontificia Universidad Católica de Valparaíso. Versión online, núm. 34, 2010, pp. 597-633. Recuperado el 27 de septiembre de 2010, de: http://www.scielo.cl/ scielo.php?pid=S0718-68512010000100019\&script $=$ sci_abstract.

${ }^{26}$ A 3 de octubre de 2010, son 139 las partes del CMNUCC que manifestaron su intención de adherirse al Acuerdo de Copenhague. Vid. UNITED NATIONS FRAMEWORK CONVENTION ON CLIMATE CHANGE. Comunicaciones recibidas de las partes en relación con la inclusión en el preámbulo del Acuerdo de Copenhague. Recuperado el 25 de septiembre de 2010, de: http://unfccc.int/meetings/items/ 5276.php.

27 Después de que la Conferencia de las Naciones Unidas sobre el Medio Ambiente y el Desarrollo celebrada en Río de Janeiro, Brasil, en 1992 acreditara a un número sin precedentes de participantes no gubernamentales (2.400 representantes de organizaciones no gubernamentales, mas de 8.000 periodistas, y 1.7000 personas que asistieron al foro de las organizaciones no gubernamentales realizado paralelamente), lo que promovió la participación de actores civiles en asuntos internacionales, la Conferencia celebrada en abril del 2010 en Cochabamba, Bolivia, logró reunir a más de 35.000 personas de 140 países, lo que la convirtió en uno de los mayores encuentros civiles de las últimas décadas. Una lista de las 241 organizaciones que se adhirieron a la convocatoria de la Conferencia puede verse en http://cmpcc.org/2010/05/31/pronunciamiento-mundial-de-los-pueblos/\#more-2061. Última visita, 23 de septiembre de 2010.
} 
Mundial de los Pueblos sobre el Cambio Climático y los Derechos de la Madre Tierra (en adelante, CMPCC) ${ }^{28}$.

Desde una perspectiva ética y ecológica basada en la protección del medio ambiente y la "Madre Tierra" ${ }^{29}$, esta conferencia congregó a diversos representantes de pueblos indígenas, comunidades campesinas, movimientos sociales, organizaciones no gubernamentales y grupos científicos y académicos con la finalidad de realizar una reflexión profunda sobre la crítica situación en la que se encuentra el planeta Tierra. Esta iniciativa partió del reconocimiento de algunas necesidades imperiosas que la humanidad debe tener en cuenta, como el respeto de los derechos de la Madre Tierra, la lucha por la justicia climática, el restablecimiento de la armonía con la naturaleza, la realización de acciones urgentes para evitar mayores daños y la elaboración de estrategias necesarias para frenar la crisis climática.

\footnotetext{
${ }^{28}$ Realizada entre el 19 y el 22 de abril de 2010 en Cochabamba, Bolivia. Dicha reunión fue convocada por el Gobierno de Bolivia al término de la Conferencia de Copenhague bajo el argumento de que el resultado de esta conferencia había sido un fracaso rotundo, y que, por lo tanto, era la sociedad la que debía brindar una respuesta paralela a la dada durante la COP15. Vid. Convocatoria de la Conferencia Mundial de los Pueblos sobre el Cambio Climático y los Derechos de la Madre Tierra de 5 de enero de 2010. Recuperado el 23 de septiembre de 2010, de: http://cmpcc.org/2010/01/07/convocatoria/\#more-1.
}

${ }^{29}$ Como resultado de la propuesta presentada por el presidente de Bolivia a la Asamblea General de las Naciones Unidas para declarar el 22 de abril como Día Internacional de la Madre Tierra, dicha organización aprobó la propuesta por aclamación de los 192 países que la integran. Vid. ASAMBLEA GENERAL DE LAS NACIONES UNIDAS, Resolución A/RES/63/278 de 1 de mayo de 2009, relativa al "Día Internacional de la Madre Tierra". Recuperado el 27 de septiembre de 2010, de: http://www.un.org/ es/comun/docs/?symbol=A/RES/63/278.

En esta ocasión, el presidente boliviano planteó que "para vivir en armonía con la naturaleza debemos reconocer que no solo los seres humanos tenemos derechos, sino también el planeta, los animales, las plantas y todos los seres vivos tienen derechos que debemos respetar". Vid. Discurso del presidente de Bolivia, Evo Morales Ayma, en ocasión de la Declaración del Día Internacional de la Madre Tierra en la Asamblea General de la ONU, Nueva York, 22 de abril de 2009. Recuperado el 26 de septiembre de 2010, de: http://cmpcc.org/derechos-madre-tierra/.

Más tarde, como resultado de la CMPCC, el presidente Evo Morales presentó nuevamente a la ONU la propuesta de creación de la Declaración Universal de los Derechos de la Madre Tierra, la cual se fundamenta en cuatro pilares básicos: el derecho a la vida de los ecosistemas, el derecho a la regeneración de la biodiversidad, el derecho a vivir sin contaminación y el derecho a la convivencia armónica con la naturaleza. Vid. Doc. FCCC/AWGLCA/2010/MISC.2 de 30 de abril de 2010, pp. 36-39. Recuperado el 27 de septiembre de 2010, de: http://unfccc.int/resource/docs/2010/awglca10/eng/misc02.pdf. 
Durante esta conferencia se organizaron 17 mesas de trabajo que abordaron distintas temáticas ${ }^{30}$; así, por ejemplo, se condenó el capitalismo como sistema consumista que estimula que se dañe al planeta; se afirmó que las emisiones de GEI provienen principalmente de los países desarrollados, quienes tienen una deuda climática con el planeta, y se proclamó la celebración de un referéndum popular mundial sobre los efectos del cambio climático.

Asimismo, durante la CMPCC se criticó fuertemente al Fondo Prototipo de Carbono controlado por el Banco Mundial ${ }^{31}$. Los participantes de la Conferencia señalaron que este fondo permite que esferas externas controlen la distribución del dinero obtenido para la implementación de los mecanismos de cumplimiento del Protocolo de Kioto ${ }^{32}$, como el Programa de Reducción de Emisiones causadas por la Deforestación

y la Degradación (REDD, por sus siglas en inglés) ${ }^{33}$, y del mecanismo para un

30 Las temáticas de los 17 grupos de trabajo de la CMPCC fueron las siguientes: causas estructurales; armonía con la naturaleza; derechos de la Madre Tierra; referéndum climático; Tribunal de Justicia Climática; migrantes climáticos; pueblos indígenas; deuda climática; visión compartida; Protocolo de Kioto; adaptación; financiamiento; desarrollo y transferencia de tecnología; bosques; peligros del mercado de carbono; agricultura y soberanía alimentaria; y estrategias de acción. Vid. CONFERENCIA MUNDIAL DE LOS PUEBLOS SOBRE EL CAMBIO CLIMÁTICO Y LOS DERECHOS DE LA MADRE TIERRA. Conclusiones finales de los grupos de trabajo de la CMPCC de 28 de abril de 2010. Recuperado el 23 de septiembre de 2010, de: http://cmpcc.org/category/grupos-de-trabajo/.

31 El Fondo Prototipo Carbono, constituido por el Banco Mundial en el año 2000, está destinado a la financiación de proyectos tendentes a reducir las emisiones de GEI en países en desarrollo por medio de la instrumentación de los mecanismos establecidos en el Protocolo de Kioto. Vid. THE WORLD BANK, Prototype carbon fund - Annual Report, Washington, DC, 2004, pp.13-14. Recuperado el 26 de septiembre de 2010, de: http://wbcarbonfinance.org/docs/2004PCFAR.pdf.

32 Vid. KIESSLING, C., Crisis ambiental global: COP 15 ¿éxito o fracaso? Análisis y prospectivas futuras, op. cit., pp. 18-21.

${ }^{33}$ Reducing Emissions from Deforestation and Degradation (REDD, por sus siglas en inglés) es el Programa de Reducción de Emisiones causadas por la Deforestación y la Degradación presentado en el 2008 por la Organización de las Naciones Unidas con el apoyo del gobierno de Noruega. Véase el portal oficial del programa en http://www.un-redd.org. Última visita, 20 de septiembre de 2010.

Durante la CMPCC, haciendo referencia al programa REDD, se señaló como prioridad que cualquier programa relacionado con los bosques debía contemplarse desde las políticas internas de los países en desarrollo. De esta manera, el grupo de trabajo 14, relativo a los bosques, concluyó que: “[...] 7) Se exige la recuperación de bosques nativos degradados, sin la intervención de acciones relacionadas con el capitalismo, o el fomento de acciones perversas encubiertas, como son las plantaciones forestales llevadas a cabo por entidades privadas dirigidas al mercado de carbono (ONG, fundaciones, institutos, transnacionales)". Vid. CONFERENCIA MUNDIAL DE LOS PUEBLOS SOBRE EL CAMBIO CLIMÁTICO Y LOS DERECHOS DE LA MADRE TIERRA. Texto de conclusiones del grupo de trabajo 14 de la CMPCC de 28 de abril de 2010. Recuperado el 23 de septiembre de 2010, de: http:// cmpcc.org/2010/04/28/conclusiones-finales-grupo-de-trabajo-14-bosques/\#more-1860. 
desarrollo limpio ${ }^{34}$, lo que provoca, en consecuencia, enormes perjuicios a los pueblos indígenas.

Después de varias reuniones en distintos escenarios, los participantes de la CMPC, como respuesta a la idea de justicia climática frente a las insatisfactorias negociaciones sobre el clima que se habían llevado a cabo y en oposición al Acuerdo de Copenhague, finalmente suscribieron un documento que contiene las conclusiones alcanzadas por los 17 grupos de trabajo: el Acuerdo de los Pueblos ${ }^{35}$.

Todo el paquete de propuestas generadas en Cochabamba fueron presentadas en abril del 2010 a la Asamblea General de las Naciones Unidas y, aunque muchas de ellas no fueron consideradas durante las primeras reuniones preliminares para la próxima COP16, algunas otras fueron incorporadas en los textos de negociación discutidos en la última reunión preparatoria realizada en Tianjin, $\mathrm{China}^{36} 37$.

Entre las principales conclusiones de la CMPCC destacan, entre otras: a) la reducción en un $50 \%$ de las emisiones de GEI por parte de los países desarrollados para el segundo período de compromisos del Protocolo de Kioto del año 2013-2017, en el cual los países desarrollados deben comprometer reducciones domésticas significativas de al menos el 50\% respecto al año base de 1990; b) la estabilización del incremento de la temperatura a $1{ }^{\circ} \mathrm{C}$ y de la tasa de 300 partes por millón de dióxido de carbono en la atmósfera; c) la distribución equitativa del espacio atmosférico, considerando la deuda climática de los países desarrollados; d) el respeto a los derechos humanos y a los

\footnotetext{
34 El mecanismo para un desarrollo limpio es un mecanismo de flexibilidad creado por el Protocolo de Kioto cuya característica principal y elemento diferenciador radica en el hecho de ser el único de los mecanismos que permite la participación de países en desarrollo en acciones de mitigación de emisiones de GEI. Para un mayor análisis de este mecanismo, véase MEJÍA, C., "Mecanismo de Desarrollo Limpio: Una debilidad convertida en oportunidad", en Pensamiento y gestión: Revista de la División de Ciencias Administrativas de la Universidad del Norte, núm. 18, Colombia, 2005, pp. 138-139; WERKSMAN, J., "The Clean Development Mechanisms: Unwrapping the "Kyoto Surprise", en Review of European Community and International Environmental Law, vol. 7, issue 2, 1998, pp. 147-158; LAFFERRIERE, R., El mecanismo de Desarrollo Limpio del Protocolo de Kyoto. Ed. CO2- EcoConsulting, Argentina, 2008 .

${ }^{35}$ Una lectura completa de las conclusiones de los 17 grupos de trabajo y el Acuerdo de los Pueblos alcanzado en la CMPCC puede realizarse en el portal oficial de la CMPCC en http://cmpcc.org/.

36 Vid. Doc. FCCC/KP/AWG/2010/17 de 5 de noviembre de 2010. Recuperado el 30 de noviembre de 2010, de: http://unfccc.int/resource/docs/2010/awg15/spa/17s.pdf.
}

37 Vid. Doc. FCCC/AWGLCA/2010/14 de 13 de agosto de 2010. Recuperado el 30 de noviembre de 2010, de: http://unfccc.int/resource/docs/2010/awglca12/spa/14s.pdf. 
derechos inherentes de los pueblos indígenas, las mujeres, los niños y los migrantes; e) el pleno reconocimiento a la Declaración de las Naciones Unidas de los Derechos de los Pueblos Indígenas; f) el reconocimiento y la defensa de los derechos de la Madre Tierra para alcanzar la armonía con la naturaleza; g) la constitución de un tribunal internacional de justicia climática que garantice el cumplimiento de los compromisos asumidos por los países desarrollados; h) el rechazo a nuevos mecanismos usados dentro del mercado de carbono que solo traspasan responsabilidades de reducción de los GEI a los países en desarrollo; i) el manejo integral de los bosques, para la mitigación y adaptación sin mecanismo de mercado, que garantice la participación de los pueblos indígenas y las comunidades locales; y j) la prohibición de conversión de los bosques naturales en plantaciones de monocultivos.

Si bien los resultados obtenidos durante la CMPCC cuentan con el apoyo de varios países en desarrollo, organizaciones no gubernamentales y gran parte de la sociedad civil, que exigen su prevalencia respecto al Acuerdo de Copenhague, en algunos casos se trata de iniciativas irrealizables o que difícilmente serán aceptadas por los países desarrollados, por lo que se convierte únicamente en una llamada a la ética y moral de cada uno de estos ${ }^{38}$.

Las conclusiones de la CMPCC que fueron escuchadas y recibidas por las Naciones Unidas no tienen un efecto vinculante, y la pretensión de exigir su incorporación y aprobación total en los textos de negociación para la COP16 podría entorpecer el desarrollo de las negociaciones. La razón principal es que a los participantes de esta conferencia les interesará debatir temas relacionados con aspectos científicos, tecnológicos y financieros, pero no sobre temas éticos como la responsabilidad moral de los países desarrollados hacia grupos humanos vulnerables como los pueblos indígenas.

De cualquier modo, los esfuerzos e intereses de la sociedad civil internacional, en especial de aquellos sectores de la población más afectados por las consecuencias del cambio climático, seguirán incrementándose en este camino paralelo al de las negociaciones climáticas intergubernamentales. Las propuestas de las reuniones no gubernamentales buscarán influir en todos los espacios de debate que se generen en

38 Vid. BBC MUNDO (22 de abril de 2010) "Un clima polarizado: Cochabamba y Copenhague". Recuperado el 29 de septiembre de 2010, de: http://www.bbc.co.uk/mundo/ciencia_tecnologia/ 2010/04/100422_2144_internacional_cambio_climatico_bolivia_cumbre_copenhague_fp.shtml. 
torno al cambio climático y, al mismo tiempo, pretenderán obligar a los Estados a desarrollar estrategias claras de lucha contra este fenómeno, aunque serán los resultados de la próxima Conferencia de Cancún los que permitirán apreciar su consideración e inclusión final.

\section{DESDE BONN A TIANJIN, ¿CUÁL ES EL ESCENARIO PARA CANCÚN?}

A lo largo del 2010 y después del fracaso de Copenhague, las partes del CMNUCC empezaron una tarea maratoniana para alcanzar un acuerdo vinculante que otorgue continuidad al Protocolo de Kioto a partir del 2012.

En este sentido, en el marco del CMNUCC y el Protocolo de Kioto, los grupos de trabajo especial establecidos en la COP11 y la COP13, a saber, el grupo de trabajo especial sobre los futuros compromisos de las partes del anexo I con arreglo al Protocolo de Kioto (en adelante, GTE-PK) ${ }^{39}$ y el grupo de trabajo especial sobre la cooperación a largo plazo en el marco del Convenio Marco de Naciones Unidas sobre el Cambio Climático (en adelante, GTE-CLP) ${ }^{40}$, se reunieron en cuatro períodos de sesiones - tres en Bonn, Alemania, y la última en Tianjin, China ${ }^{41}$ - a fin de llevar a cabo las reuniones preparatorias para la próxima Conferencia de las Partes, y posteriormente informar de los resultados de su trabajo a la XVI Conferencia de las

\footnotetext{
${ }^{39}$ Durante la XI Conferencia de las Partes del CMNUCC (COP11) y el primer período de sesiones de la Conferencia de las Partes - que sirvió como reunión de las partes del Protocolo de Kioto- (COP-RP1), que tuvo lugar en el 2005 en Montreal, Canadá, se estableció el GTE-PK en base al artículo 3.9 del Protocolo, que dispone la consideración de nuevos compromisos para los Estados integrados en el anexo I al menos siete años antes del final del primer período de compromisos (2012).

${ }^{40}$ Durante la XIII Conferencia de las Partes del CMNUCC (COP13) y el tercer período de sesiones de la Conferencia de las Partes — que sirvió como reunión de las partes del Protocolo de Kioto- (COP-RP3), que se desarrolló en diciembre del 2007 en Bali, Indonesia, se adoptó el Plan de Acción de Bali, que estableció el GTE-CLP con la facultad de desarrollar elementos clave para la cooperación a largo plazo en ámbitos como: mitigación, adaptación, financiamiento y transferencia de tecnologías.

${ }^{41}$ La información relativa a las reuniones preparatorias para la COP16 se encuentra disponible en el portal oficial del CMNUCC: http://unfccc.int/meetings/intersessional/bonn_10_2/items/5692.php. Última visita, 7 de octubre de 2010 .
} 
Naciones Unidas sobre el Cambio Climático, que tendrá lugar en Cancún, México, del 29 de noviembre al 10 de diciembre de $2010^{42}$.

En estas reuniones, el GTE-PK, el GTE-CLP, representantes de gobiernos y organizaciones no gubernamentales, académicos y representantesdel sector privado y de los medios de comunicación discutieron —organizados en distintos espacios de debate — los aspectos necesarios para encontrar soluciones al caos climático.

Las negociaciones comenzaron en abril del 2010 por la presión de la CMPCC sobre los mandatarios de los países desarrollados. Del 9 al 11 de abril en Bonn, Alemania, el GTE-CLP y el GTE-PK tuvieron sus primeras reuniones preparatorias del $2010 \mathrm{con}$ el fin de recobrar la confianza que se había perdido en Copenhague. Algunos representantes de países miembros del CMNUCC incluso se mostraron interesados en acordar la firma, hasta fin de año, de un acuerdo vinculante de reducción de gases contaminantes que pudiera sustituir al Protocolo de Kioto $^{43}$.

\footnotetext{
${ }^{42}$ Cabe mencionar que, para la celebración de la COP15, hubo varios períodos de reuniones de los dos grupos especiales (GTE-CLP y GET-PK). Durante el 2008 hubo cuatro períodos de sesiones: en abril en Bangkok, Tailandia; en junio en Bonn, Alemania; en agosto en Accra, Ghana; y en diciembre en Poznan, Polonia. En el 2009, los grupos de trabajo especial se reunieron en abril, junio y agosto en Bonn, Alemania; en octubre en Bangkok, Tailandia; en noviembre en Barcelona, España; y en diciembre en Copenhague, Dinamarca.
}

Durante las reuniones del 2009, el GTE-CLP centró su trabajo en el desarrollo de un nuevo texto de negociación que al final de las reuniones resultó ser un documento de aproximadamente 200 páginas. Dado que se trataba de un texto excesivamente largo, los participantes de las reuniones desarrollaron paralelamente documentos no oficiales que fueron enviados como anexos del informe final del grupo a Copenhague, situación que perjudicó el desarrollo de las negociaciones en la COP15.

Por su parte, el GTE-PK durante el 2009 concentró su trabajo en la especificación de las metas de reducción de emisiones de GEI de las partes del anexo I del Protocolo de Kioto para después del 2012 y en el análisis de aspectos relacionados con los mecanismos de flexibilidad y el uso de la tierra, cambio en el uso de la tierra y silvicultura. Sin embargo, el debate en este grupo estuvo dividido; mientras que algunos proponían la enmienda del Protocolo de Kioto en base al artículo 3.9 de dicho acuerdo, referido a nuevos compromisos de las partes, otros preferían el análisis de temas con menor grado de complejidad, como los mecanismos de flexibilidad y el uso y cambio de uso de la tierra. Las reuniones de este grupo desataron una serie de desacuerdos entre los participantes, que discutieron la posibilidad de que en la COP15 se logre una enmienda al Protocolo de Kioto o, por el contrario, se establezca un nuevo acuerdo individual que reúna el trabajo del GTE-CLP y el GTE-PK.

Finalmente, el resultado de la COP15 parece mostrar que el trabajo realizado por los dos grupos especiales a lo largo del 2008 y el 2009 solo sirvió para que los países desarrollados suscribieran un documento que no solo no consideró a los países en desarrollo, sino que también fue firmado sin el apoyo del propio CMNUCC.

${ }^{43}$ Un informe del noveno período de sesiones del grupo de trabajo especial de cooperación a largo plazo en el marco del Convenio sobre el Cambio Climático y el undécimo período de sesiones del grupo de trabajo especial sobre los nuevos compromisos de las partes del anexo I con arreglo al Protocolo de Kioto se puede consultar en INTERNATIONAL INSTITUTE FOR SUSTAINABLE DEVELOPMENT (ISD), "Sumary of the Bonn Climate Change Talks: 9-11 april 2010", en Earth Negotiations Bulletin, vol. 12, núm. 460, 2010. Recuperado el 25 de septiembre 2010 de: http://www.iisd.ca/download/pdf/ enb12460e.pdf (25/10/2010). 
Si bien el interés de los países desarrollados por dar plena validez al documento acordado en la COP15 era evidente, los países miembros del Grupo de los 77 (en adelante, G-77) y China ${ }^{44}$ apoyaron la propuesta de mantener la vigencia del Protocolo de Kioto si su substituto era el Acuerdo de Copenhague. Aunque la mayoría de los países en desarrollo exigían el cumplimiento de los compromisos de reducción de GEI establecidos en el Protocolo de Kioto a fin de evitar el incremento de la temperatura mundial más allá de $1,5^{\circ} \mathrm{C}$ hasta el 2050 , países como Colombia y los de la Alianza de Pequeños Estados Insulares (AOSIS, por sus siglas en inglés) ${ }^{45}$, ante la imposibilidad de crear un nuevo documento jurídicamente vinculante, defendieron la posibilidad de mantener vigente el texto generado en Copenhague, lo que dio lugar a los primeros desacuerdos entre los participantes.

Algunos representantes de países desarrollados decidieron debatir la permanencia del Acuerdo de Copenhague como un documento que debía ser aceptado por todas las partes del CMNUCC; sin embargo, dadas las dificultades presentadas en las sesiones y ante el temor de reproducir errores pasados, finalmente se optó por analizar temas procedimentales, razón por la que durante los tres días de reunión se analizaron aspectos relativos a la organización y los métodos de trabajo para las siguientes reuniones preparatorias.

Al final de este primer período de sesiones, los delegados del GTE-CLP ordenaron a su presidente la elaboración de un texto de negociación para el período de sesiones que se desarrollarían en junio del 2010, y el GTE-PK acordó que en las siguientes reuniones, que se desarrollarían a lo largo del mismo año, continuarían considerando las reducciones de emisiones conjuntas e individuales de las partes del anexo I del Protocolo de Kioto.

\footnotetext{
${ }^{44}$ El Grupo de los 77 (G-77) fue creado el 15 de junio de 1964 por 77 países en desarrollo, que firmaron la Declaración Conjunta de los Setenta y Siete Países, emitida al finalizar el primer período de sesiones de la Conferencia de las Naciones Unidas sobre Comercio y Desarrollo (UNCTAD), que se celebró en Ginebra. Aunque el número de países miembros del G-77 asciende actualmente a 132, se ha mantenido el nombre original a causa de su importancia histórica. Para mayor información sobre el G-77, véase el portal oficial del grupo en http://www.g77-doha.org/espanol/general_info.htm.

${ }^{45}$ La Alliance of Small Island States o Alianza de Pequeños Estados Insulares es una coalición de 43 países insulares de tierras bajas y pequeñas dimensiones, en su mayoría miembros del G-77, que son particularmente vulnerables a la subida del nivel del mar. Los países de la AOSIS están unidos por la amenaza que el cambio climático representa para su supervivencia, y frecuentemente adoptan una postura común en las negociaciones.
} 
Con este primer antecedente, las discusiones prosiguieron en Bonn, Alemania, entre el 31 de mayo y el 11 de junio de 2010, fechas en las que tuvo lugar la 32. ${ }^{a}$ reunión de los órganos subsidiarios del CMNUCC, el décimo período de sesiones del GTE-CLP y el duodécimo período de sesiones del GTE-PK.

En esta segunda ronda de reuniones preparatorias varios fueron los aspectos que se pretendían discutir, aunque poco el tiempo y divergentes los intereses. Los representantes de los 185 países asistentes pretendían analizar aquellos aspectos que quedaron pendientes en la COP15. El GTE-CLP discutió el proyecto de texto que había sido diseñado por su presidenta, Margaret Mukahanana-Sangarwe, de Zimbabwe, y que fue distribuido entre todos los asistentes ${ }^{46}$; y el GTE-PK centró su atención en las reducciones de emisiones del anexo I y los mecanismos de flexibilidad.

Las reuniones del GTE-CLP se vieron obstaculizadas por los desacuerdos y las críticas que mereció el texto de negociación puesto a consideración de los participantes. Dicho texto debía recoger las aportaciones y propuestas de los países desarrollados y países en desarrollo en relación con las posibles soluciones para el cambio climático; sin embargo, estas propuestas, principalmente de los países en desarrollo, no fueron incorporadas en su contenido, lo que provocó el rechazo de algunos representantes, que incluso lo calificaron como un "Copenhagen Accord Plus"47.

Mientras que algunos países desarrollados pretendían obstaculizar las negociaciones oponiéndose a la inclusión de temas importantes como la consideración de $1{ }^{\circ} \mathrm{C}$ o $1,5^{\circ} \mathrm{C}$ como nivel máximo de incremento de temperatura, o la limitación del consumo de

\footnotetext{
${ }^{46}$ Vid. Doc. FCCC/AWGLCA/2010/6 de 17 de mayo de 2010, recuperado el 29 de septiembre de 2010, de: http://unfccc.int/resource/docs/2010/awglca10/spa/06s.pdf.

${ }^{47}$ En el nuevo texto presentado por el GTE-CLP se omitieron algunos temas de gran importancia para las negociaciones como los aspectos relacionados con la justicia climática y la protección de los derechos de los pueblos indígenas y las comunidades campesinas. Aunque países como Chile, Panamá, República Dominicana y Costa Rica mostraron su apoyo al contenido de dicho texto, algunos representantes de otros países, como Nicaragua, afirmaron que su contenido generaba obligaciones a los países más pobres, por lo que su aceptación era inviable. Vid. CENTRO HUMBOLDT Para la promoción del desarrollo territorial y la gestión ambiental, "Finaliza Bonn II con un Copenhagen Accord Plus". Recuperado el 3 de octubre de 2010, de: http://www.humboldt.org.ni/blog/2010/06/14/finaliza-bonn-ii-con-un-copenhaguenaccord-plus.
} 
energías $^{48}$, los países del G-77 y China denunciaron que el texto base del GTE-CLP, al no incluir las propuestas de los países en desarrollo, no garantizaba su efectiva, transparente y democrática participación en las reuniones intergubernamentales ${ }^{49}$. Igualmente, señalaron que, a diferencia del Protocolo de Kioto, el Acuerdo de Copenhague no establece compromisos obligatorios, por lo que aceptar la legalidad de dicho acuerdo, tal como había manifestado el propio representante de Nicaragua, sería "quitarle la vida al Protocolo de Kioto"s0.

Aunque en este período de sesiones se pretendía discutir la permanencia del documento elaborado en diciembre del 2009, los participantes prefirieron analizar otros temas adicionales como el referido a la financiación. En esta ocasión, algunos países desarrollados —incluida Europa, que atravesaba momentos difíciles en su economía ${ }^{51}$ anunciaron el incremento de su ayuda a corto plazo para los países en desarrollo. Esta disponibilidad anunciada por los países desarrollados significó un paso adelante en las negociaciones; no obstante, cuando se analizaron otros aspectos, como la creación de un

${ }^{48}$ Los Estados Unidos, Arabia Saudí, Omán, Kuwait y Qatar fueron los ganadores de los "Fossil of the day" entregados el 5 y 8 de junio por The Climate Action Network (CAN), una coalición de más de 500 ONG de todo el mundo que entregan estos premios a los países que obstaculizan el proceso de negociación. Vid. CAN International, "Fossil of the day", Bonn, Alemania, 2-2010. Recuperado el 28 de septiembre de 2010, de: http://www.climatenetwork.org/event/bonn-2-2010.

49 Los representantes de países como Bolivia, Argentina, Nicaragua, Ecuador y Venezuela señalaron que la primera propuesta presentada por Margaret Mukahanana-Sangarwe, presidenta del GTE-CLP, era un texto unilateral que no contenía las aportaciones de los países en desarrollo, y que, por tanto, dificultaría el proceso de negociación. Para un análisis pormenorizado de la participación de los países en desarrollo en las reuniones preparatorias, véase INSTITUTO INTERNACIONAL PARA EL DESARROLLO SOSTENIBLE (IIDS), "Conversaciones de Bonn sobre el clima", en Boletín de Negociaciones de la Tierra, vol. 12, núm. 461, 2010. Recuperado el 25 de septiembre de 2010, de: http://www.iisd.ca/climate/ sb32/compilations.pdf.

50 Vid. La Razón (12 de junio de 2010), “Protocolo de Kyoto sería excluido en Cancún”. Recuperado el 22 de septiembre de 2010, de: http://www.la-razon.com/version.php?ArticleId=3356\&EditionId=104.

Es importante señalar que los países en desarrollo no están dispuestos a abandonar el Protocolo de Kioto, pues este sería el único instrumento vinculante para obligar a los países desarrollados a reducir sus emisiones de GEI. El temor después de Copenhague es que el instrumento sucesor del Protocolo no contenga compromisos que obliguen a esas reducciones y que, por el contrario, al ser demasiado laxo, olvide considerar aspectos importantes como la financiación que deberían recibir los países en desarrollo para la realización de acciones de mitigación y adaptación al cambio climático.

51 La Unión Europea prometió 2.800 millones de euros anuales por concepto de financiación, que aportaría entre los años 2010 y 2012. Por su parte, los Estados Unidos y Canadá anunciaron el incremento en la financiación en torno al cambio climático por su propia cuenta, aunque es evidente que esos fondos todavía no tienen un destinatario concreto, pues una parte podría ir, por ejemplo, a los fondos de inversión del clima del Banco Mundial, lo que generaría una mayor desconfianza en los países en desarrollo, que creen que este organismo, al financiar proyectos de carbono o explotación de bosques, es uno de los principales causantes de la crisis ambiental. Vid. INTERNATIONAL CENTRE FOR TRADE AND SUSTAINABLE DEVELOPMENT, "Year-End Climate Goal in Question as Bonn Meetings Begin", en Bridges Weekly Trade News Digest, vol. 14, núm. 20, 2010. Recuperado el 20 de septiembre de 2010, de: http://ictsd.org/i/news/puentesquincenal/77648/. 
fondo verde climático ${ }^{52}$, la división entre Estados fue evidente, pues no se pudo lograr acuerdos sobre asuntos referidos a su funcionamiento, rol y administración ${ }^{53}$.

Las cuestiones relacionadas con el Programa de Reducción de Emisiones causadas por la Deforestación y la Degradación Plus (REDD+, por sus siglas en inglés) ${ }^{54}$, la creación de un nuevo mecanismo de transferencia de tecnología, y la creación de capacidades y en cierta medida su financiación ${ }^{55}$ fueron temas que tuvieron un gran apoyo y amplios espacios de debate; sin embargo, era evidente que estos no eran los únicos aspectos que los países en desarrollo, grandes perdedores de este segundo período de reuniones preparatorias, querían debatir. Así, por ejemplo, el presidente de Bolivia manifestó su rechazo a cualquier documento que no incluyera las conclusiones de la CMPCC, pues hasta ese momento, y pese a la predisposición del secretario ejecutivo del CMNUCC a incluirlas, los textos de negociación elaborados y puestos a consideración de los participantes no contenían ninguna de las aportaciones realizadas por esta conferencia ${ }^{56}$. Las conclusiones de este segundo período de negociaciones de los grupos de trabajo especial del Convenio mostraron, una vez más, la falta de acuerdo que existe entre los

\footnotetext{
52 En septiembre del 2010 se llevó a cabo en Suiza una reunión de representantes de Estados donde 45 países intentaron poner en marcha el llamado Fondo Verde con el objetivo de recaudar unos 30.000 millones de dólares, cuyo destino serían los países más afectados por el cambio climático durante el período 2010-2012. Cabe recordar que durante las negociaciones de Copenhague los países industrializados prometieron ayudar con 30.000 millones de dólares (nuevos y adicionales) para el 2010-2012. Asimismo, 120 países en Copenhague se comprometieron a aumentar su ayuda para el desarrollo de las naciones más empobrecidas a 100.000 millones de dólares a partir del 2020. Vid. Acuerdo de Copenhague, cit.

53 Los países en desarrollo solicitaron que los países industrializados comprometieran a favor de este fondo el $1,5 \%$ de su PIB y establecieran las funciones de monitorización, información y verificación de este. Vid. INSTITUTO INTERNACIONAL PARA EL DESARROLLO SOSTENIBLE (IIDS), "Conversaciones de Bonn sobre el clima", op. cit. p. 12.

${ }^{54} \mathrm{El}$ programa Reducing emissions from deforestation and forest degradation "plus" conservation, the sustainable management of forests and enhancement of forest carbon stocks (REDD+, por sus siglas en inglés) se refiere a proyectos de reducción de emisiones que incluyen, además, acciones y proyectos para "el manejo sostenible de los bosques, la conservación forestal, la reforestación y el incremento de las reservas de carbono forestales". REDD+ podría incluir no solamente los bosques, sino también la agricultura, los suelos y el biocarbón. Los críticos de este programa señalan que incluir la agricultura y los suelos en su desarrollo alienta la conversión de grandes espacios en mercancía, mientras que la inclusión del biocarbón podría aumentar la expansión de plantaciones forestales. Vid. AMIGOS DE LA TIERRA BRASIL. REDD y el futuro de los bosques. Porto Alegre, Brasil, 2007. Recuperado el 5 de octubre de 2010, de: http://www.natbrasil.org.br/Docs/publicacoes/cartilhareddweb.pdf.
}

${ }^{5}$ Vid. INTERNATIONAL CENTRE FOR TRADE AND SUSTAINABLE DEVELOPMENT, "Year-End Climate Goal in Question as Bonn Meetings Begin", op. cit.

56 Vid. Los Tiempos (4 de julio de 2010), "Las negociaciones de Bonn y el cambio climático". Recuperado el 27 de septiembre de 2010, de: http:/www.lostiempos.com/oh/actualidad/actualidad/ 20100704/las-negociaciones-de-bonn-y-el-cambio-climatico_78283_147654.html. 
países en desarrollo y los países desarrollados. En un ambiente lleno de incertidumbre, se empezó a contemplar la posibilidad de que algunas alternativas que nacieron a raíz de la firma del Protocolo de Kioto, como la venta de "aire caliente"57 o la utilización de los mercados de carbono para "compensar" las emisiones, podrían ser algunas de las salidas que los países desarrollados utilizarían para evitar cumplir los compromisos de recortar sus emisiones en un $30 \%$ y un $40 \%$ para el 2020 , lo que dificultaría el escenario para la posible suscripción de un acuerdo vinculante.

Después de las fuertes críticas que se emitieron en relación con la deficiente tarea de los grupos de trabajo especial del CMNUCC durante el segundo período de reuniones, del 2 al 6 agosto de 2010 tuvo lugar el tercer período de reuniones preparatorias para la COP16 en Bonn, Alemania.

Dado que la mayoría de los países en desarrollo rechazaron la primera versión del texto de negociación del GTE-CLP debido a que, entre otras cosas, establecía responsabilidades desproporcionadas entre los países y no incorporaba las prioridades de los sectores más vulnerables del planeta, el nuevo documento que fue distribuido en julio del 2010, así como las propuestas presentadas por el presidente del GTE-PK, mostraron avances importantes ${ }^{58}$.

El propósito de este tercer período de reuniones preparatorias del GTE-CLP era obtener un texto de negociación que facilitara las negociaciones en la COP16; sin embargo, se pusieron en la mesa de discusión algunos temas adicionales que generaron nuevas discrepancias entre los participantes y, con ello, mayores retrasos en el proceso. Así, por

\footnotetext{
${ }^{57}$ El denominado "Hot Air" o "aire caliente" es el número sobrante de derechos del que dispondrían los países del grupo de las economías en transición (sobre todo Europa del Este y Rusia), básicamente por haber sufrido un importante descenso de la actividad económica desde 1990, lo cual les permitiría vender sus derechos a países que los hubieran superado y que los necesitan para cumplir sus compromisos de reducción de emisiones. El comercio de emisiones no es lo mismo que el comercio de "aire caliente", pero el "aire caliente" solo puede ser comercializado con el comercio de emisiones, aunque es evidente que, sin el comercio de "aire caliente", la reducción de emisiones podría ser mucho más baja que la fijada como objetivo inicial, por lo que, en consecuencia, es un problema ambiental. Sobre el mercado de "aire caliente" puede verse WOERDMAN, E., "Hot Air Trading under the Kyoto Protocol: An Environmental Problem or Not?", en European Environmental Law Review, vol. 14, 2005, pp. 71-77. Recuperado el 10 de septiembre de 2010, de: http://rechten.eldoc.ub.rug.nl/FILES/root/Algemeen/Recht13/2005/ hotairtrading/HotAirTrading_EELR.PDF.

${ }^{58}$ Vid. Doc. FCCC/AWGLCA/2010/8 de 9 de julio de 2010. Recuperado el 28 de septiembre de 2010, de: http://unfccc.int/documentation/documents/advanced_search/items/3594.php?rec=j\&priref=600005899; y Doc. FCCC/KP/AWG/2010/CRP2 de 6 de agosto de 2010. Recuperado el 28 de septiembre de 2010, de: http://unfccc.int/documentation/documents/advanced_search/items/3594.php?such=j\&symbol=FCCC/ $\mathrm{KP} / \mathrm{AWG} / 2010 / \mathrm{CRP} .2$.
} 
ejemplo, si bien se obtuvieron avances sobre las reglas técnicas de los mecanismos de flexibilidad, se discutieron de nuevo aspectos relacionados con el programa REDD+. Durante la COP15 muchas de las características de este programa fueron discutidas por los países desarrollados sin la participación de los países en desarrollo, lo que conllevó que durante las conversaciones en Bonn se destacaran aspectos importantes como los relativos a los mercados para la implementación del programa, la equidad en su desarrollo y su posible impacto sobre los pueblos indígenas ${ }^{59}$.

Las discusiones del GTE-PK se centraron en la consideración de la escala de las reducciones de emisiones de las partes del anexo I del Protocolo de Kioto y en cuestiones legales como la posible brecha entre el primer período de los compromisos del Protocolo y los que le sigan. Igualmente se analizaron aspectos relativos al uso de la tierra, el cambio en el uso de la tierra y la silvicultura, los mecanismos de flexibilidad y las consecuencias potenciales de las medidas de respuesta al cambio climático.

El tercer período de reuniones finalizó con pocos resultados ${ }^{60}$. Por un lado, la falta de voluntad mostrada por los participantes durante las negociaciones y, por otro, la desilusión que generó la falta de apoyo del Senado de los Estados Unidos a la propuesta de Ley de Energía y Clima presentada por el presidente Barack Obama ${ }^{61}$ — la cual, pese a no estar vinculada directamente con las negociaciones del clima, era fundamental a la hora de analizar los compromisos de reducción de GEI que los Estados Unidos podrían asumir - fueron dos aspectos que afectaron al desarrollo de las negociaciones, razón

\footnotetext{
59 Vid. INSTITUTO INTERNACIONAL PARA EL DESARROLLO SOSTENIBLE (IIDS), "Síntesis de las conversaciones de Bonn sobre el cambio climático: 2 al 6 de agosto de 2010", en Boletín de Negociaciones de la Tierra, vol. 12, núm. 478, 2010. Recuperado el 1 de octubre de 2010, de: http:// www.iisd.ca/download/pdf/enb12478s.pdf.

60 Vid. EUROPA PRESS (6 de agosto de 2010), "Finaliza la reunión de la ONU sobre el cambio climático, con más retrocesos que avances". Recuperado el 21 de septiembre de 2010, de: http:// www.europapress.es/internacional/noticia-finaliza-reunion-onu-cambio-climatico-mas-retrocesosavances-20100806230411.html.

61 La aprobación de la Ley de Energía y Clima propuesta por el presidente Obama al Senado de los Estados Unidos suponía para la comunidad internacional una vía para que el resto de los países desarrollados pudieran asumir sus compromisos vinculantes, pues con esta ley los Estados Unidos debían presentar objetivos concretos de reducción de emisiones. Sin embargo, esta ley fue desestimada por el Senado por considerar que no era posible la aprobación de una norma legal que establezca reducciones de emisiones de gases contaminantes, con lo que salió a relucir nuevamente la estrecha relación que aún existe entre las grandes empresas petroleras y algunos de los representantes de aquel país. Este veto legal supuso la primera gran derrota del presidente Obama, quien durante Copenhague se había comprometido a una reducción del 17\% de los gases contaminantes. Vid. El Mundo (23 de julio de 2010), "EEUU no tendrá ley contra el cambio climático". Recuperado el 1 de octubre de 2010, de: http://www.elmundo.es/ america/2010/07/23/estados_unidos/1279920449.html.
} 
por la cual la atención se centraría en el último período de reuniones preparatorias para la COP16.

La última ronda de reuniones preparatorias del GTE-PK y el GTE-CLP tuvo lugar del 4 al 9 de octubre de 2010 en Tianjin, China. Cabe recordar que desde las primeras reuniones preparatorias celebradas después de Copenhague, y debido a que no solo se buscaba obtener un documento de limitación o reducción de emisiones sino un acuerdo que lograra una transformación social y económica de los Estados, la división de intereses en las negociaciones ha sido un obstáculo en el desarrollo y fortalecimiento del régimen jurídico del cambio climático. Esta división fue evidente una vez más en las reuniones de Tianjin, donde los participantes no lograron aclarar el complejo proceso de las negociaciones.

En el caso del GTE-PK se analizó la propuesta de su presidente, que había sido puesta a consideración de los participantes en el mes de agosto. En esta propuesta se incluían temas como la posible enmienda del artículo 3.9 del Protocolo de Kioto y las mejoras de los mecanismos de flexibilidad y los proyectos de uso de la tierra, cambio del uso del suelo y silvicultura ${ }^{62}$. Aunque las partes procuraron centrar su atención en la limitación de las opciones e intentaron avanzar en las cuestiones sustanciales, no se pudieron llegar a acuerdos sobre temas importantes como la definición de nuevos compromisos de reducción de emisiones para el segundo período del Protocolo de Kioto. En la conclusión de las reuniones de este grupo se estableció que la propuesta final revisada por el presidente sería puesta a consideración de la Conferencia de las Partes en Cancún ${ }^{63}$.

Por su parte, el texto de negociación elaborado por la presidencia del GTE-CLP para su discusión y debate en Tianjin mostraba grandes avances, pues incluía algunas de las propuestas realizadas por los países en desarrollo desde el inicio de las negociaciones, como el mantenimiento del escenario de $1,5^{\circ} \mathrm{C}$ o $2{ }^{\circ} \mathrm{C}$ de incremento de temperatura, la posibilidad de constituir un tribunal de justicia climática y la financiación en beneficio

\footnotetext{
${ }^{62}$ Vid. Doc. FCCC/KP/AWG/2010/CRP.2 cit.

${ }^{63}$ Vid. Doc. FCCC/KP/AWG/2010/CRP.3 de 9 de octubre de 2010. Recuperado el 11 de octubre de 2010, de: http://unfccc.int/resource/docs/2010/awg14/eng/crp03.pdf.
} 
de los países en desarrollo para propiciar su adaptación y mitigación del cambio climático $^{64}$.

Dentro de este grupo, las discusiones se centraron básicamente en los aspectos relativos a la adaptación, la tecnología y el programa REDD+. Los países en desarrollo y los países desarrollados acordaron la necesidad de analizar otros asuntos de gran importancia, como los compromisos y las medidas de mitigación, la información, la verificación de documentación y el apoyo a los países en desarrollo, prestando especial atención al tema de la mitigación como eje central de las negociaciones en Cancún ${ }^{65}$.

Finalmente las partes acordaron que reflejarían el trabajo desarrollado en este último período de sesiones preparatorias del 2010 en un documento informativo dirigido a la Secretaría, y que el texto de negociación de la GTE-CLP quedaría como base para las negociaciones de Cancún.

Pese a las importantes inclusiones que se hicieron en los textos de negociación y aunque los participantes conocían la importancia de este último período de reuniones preparatorias para la COP16, las reuniones del GTE-CLP y el GTE-PK mostraron nuevamente la falta de acuerdo entre los países en desarrollo y los países desarrollados, sobre todo en temas tan específicos y de gran importancia como los compromisos de reducción de emisiones de GEI. Desde la perspectiva de algunos países en desarrollo, las últimas reuniones celebradas en Tianjin mostraron mejoras en el proceso de negociación, principalmente por la actitud positiva de la mayoría de participantes en las reuniones y en los debates informales; no obstante, otros elementos, como el desacuerdo entre los delegados de China y los Estados Unidos, quienes hasta el último día de debate se hicieron recíprocamente duras críticas por la falta de voluntad en el proceso de negociación ${ }^{66}$, reflejan que el escenario para Cancún no es muy alentador.

\footnotetext{
${ }^{64}$ Vid. Doc. FCCC/AWGLCA/2010/14 cit.

65 Vid. INTERNATIONAL INSTITUTE FOR SUSTAINABLE DEVELOPMENT (IISD), "Tianjin Climate Change Talks: 4-9 October 2010”, en Earth Negotiations Bulletin, vol. 12, núm. 479, 2010. Recuperado el 12 de octubre de 2010, de: http://www.iisd.ca/climate/ccwg12/compilatione.pdf.

66 Vid. BBC MUNDO (9 de octubre de 2010), "Pesimismo rodea las negociaciones sobre el Cambio Climático". Recuperado el 14 de octubre de 2010, de: http://www.bbc.co.uk/mundo/ultimas_noticias/ 2010/10/101009_ultnot_cambio_climatico_tianjin_choque_eeuu_china_aw.shtml.
} 
Con todo, tanto el proceso de negociación llevado a cabo por los grupos de trabajo especial del CMNUCC durante el 2010 — que dio lugar a unos textos de negociación que serán presentados en diciembre en Cancún ${ }^{67}$-, como la incorporación de las propuestas realizadas por la CMPCC en dichos textos ${ }^{68}$, son una muestra de que los caminos paralelos que hasta ahora han seguido las negociaciones intergubernamentales y no gubernamentales sobre el cambio climático tendrán que converger en un mismo punto de encuentro si se quiere obtener verdaderos compromisos vinculantes. E1 resultado final se verá en la COP16, donde las partes del CMNUCC no solo tendrán que discutir las propuestas presentadas, sino también encontrar soluciones definitivas al problema climático que aqueja a toda la humanidad.

\section{CONSIDERACIONES FINALES (EL RETO EN CANCÚN: UN ACUERDO JURÍDICAMENTE VINCULANTE)}

El cambio climático es una realidad y desastres como los incendios de Rusia, las inundaciones de Pakistán y China, las sequías de Níger y el Sahel, las tormentas e inundaciones en el centro y el sur de América, el deshielo del Ártico y el incremento de temperatura superficial de los océanos ${ }^{69}$ son una muestra clara de que el calentamiento atmosférico está provocando grandes pérdidas económicas, ambientales y sociales a nivel mundial.

Las negociaciones y los acuerdos relativos al cambio climático no pueden, ni deben, posponerse por más años, pues de lo contrario los daños se incrementarán y los

\footnotetext{
${ }^{67}$ Doc. FCCC/AWGLCA/2010/14 cit. y Doc. FCCC/KP/AWG/2010/17 cit.
}

68 Algunas de las propuestas de la CMPCC que fueron incluidas en los textos de negociación para la COP16 se refieren a acciones de mitigación que hagan prevalecer los derechos de la naturaleza; a la necesidad de una visión más íntegra sobre los bosques; a la limitación del incremento de temperatura en 1 ${ }^{\circ} \mathrm{C}$; a la reducción de emisiones en más de un 50\% para el 2017; a la financiación de acciones contra el cambio climático en los países en desarrollo con el 6\% del PIB de los países desarrollados; y al levantamiento de las barreras de propiedad intelectual para facilitar la transferencia de tecnología. Vid. CONFERENCIA MUNDIAL DE LOS PUEBLOS SOBRE EL CAMBIO CLIMÁTICO Y LOS DERECHOS DE LA MADRE TIERRA, "Comunicado del Estado Plurinacional de Bolivia: las propuestas de Cochabamba van a Cancún" de 10 de octubre de 2010. Recuperado el 14 de octubre de 2010, de: http://cmpcc.org/2010/10/10/comunicado-del-estado-plurinacional-de-bolivia/\#more-2397.

69 Se pueden ver los datos actualizados sobre el incremento de temperatura en la superficie terrestre y marina durante el año 2010 en NOAA, "Second Warmest July and Warmest Year-to-Date Global Temperature on Record" de 13 de agosto de 2010. Recuperado el 5 de octubre de 2010, de: http:// www.noaanews.noaa.gov/stories2010/20100813_globalstats.html. 
impactos irreversibles serán incalculables. Acelerar el ritmo de las negociaciones climáticas y convertir las palabras en acciones efectivas es sin duda una misión que deben asumir los Estados y la sociedad civil el próximo mes de diciembre en Cancún cuando tenga lugar la COP16.

El actual ambiente de desconfianza, que se inició en Copenhague y que se vio nuevamente reflejado durante las reuniones preparatorias para la COP16 realizadas en Bonn y Tianjin, debe ser un aspecto considerado por todos los participantes de la próxima conferencia sobre el cambio climático, que además tienen que ser conscientes de que la falta de acuerdos y la generación de un nuevo ambiente de desconfianza pueden provocar que las esperanzas, una vez más, se conviertan en frustraciones.

Los resultados de las cuatro reuniones preparatorias para la COP16 celebradas por el GTE-PK y el GTE-CLP del CMNUCC evidencian que las posibilidades para lograr un acuerdo jurídicamente vinculante en Cancún son cada vez menores, lo que demuestra que las negociaciones del régimen jurídico del cambio climático, una vez más, no lograrán traducirse en una batería de soluciones reales.

Las últimas reuniones preparatorias de la COP16 que se celebraron en octubre de 2010 en Tianjin, China, mostraron que los gobiernos se encuentran en la necesidad de aprobar un conjunto de medidas que demuestre su compromiso político y humano. No obstante, es importante señalar que la aprobación de este paquete de medidas o de cualquier acuerdo jurídicamente vinculante deberá sopesar muchos de los obstáculos que surgirán nuevamente en Cancún. La polarización de opiniones entre países desarrollados y países en desarrollo, o entre instancias intergubernamentales y no gubernamentales, sobre temas como la financiación o la mitigación de los impactos climáticos, la recaudación y el manejo del Fondo Verde ${ }^{70}$, o los compromisos de reducción de emisiones de los países desarrollados y de las economías emergentes, crearán un ambiente complejo en las negociaciones.

\footnotetext{
${ }^{70}$ En septiembre del 2010 el gobierno de los Países Bajos creó una página de Internet denominada "Fast Start Finance" con el propósito de hacer el seguimiento de las aportaciones de los países desarrollados al Fondo Verde. Los programas y el monto de dinero ofrecidos por determinados países desarrollados como Dinamarca, Francia, Alemania o el Reino Unido pueden verse en el portal oficial de Fast Start Finance en http://www.faststartfinance.org. Última visita, 3 de octubre de 2010.
} 
En diciembre se discutirán las conclusiones a las que lleguen los grupos de trabajo especial del CMNUCC, las cuales incluirán en los textos de negociación las propuestas salidas de las reuniones intergubernamentales y no gubernamentales en torno al cambio climático. Los participantes de la COP16, desde su diversidad de intereses, pero bajo un mismo objetivo, tendrán que decidir entre completar los trabajos llevados a cabo en Copenhague, obtener un nuevo instrumento jurídicamente vinculante, o, en el peor de los escenarios, sentar las bases para la próxima conferencia sobre el cambio climático que se celebrará en el 2011 en Sudáfrica; sin embargo, lo único claro es que el fracaso no es una opción, y que los Estados deben trabajar con un renovado sentido de urgencia y flexibilidad para acordar soluciones climáticas conjuntas que reparen el daño que se ha provocado al planeta y que las futuras generaciones observarán y juzgarán.

\section{BIBLIOGRAFÍA}

\section{Referencias doctrinales}

ESTRADA, R., “Acuerdo de Copenhague: las negociaciones sobre el cambio climático después de la XV Conferencia”, en Revista del Centro de Economía Internacional, núm. 17, 2010, p. 114-115. Recuperado el 22 de septiembre de 2010, de: http:// www.cei.gov.ar/revista/17/parte5b.pdf.

KIESSLING, C., Crisis ambiental global: COP 15 ¿éxito o fracaso? Análisis y prospectivas futuras, Ed. FLACSO, Argentina, 2010, p. 4. Recuperado el 23 de septiembre de 2010, de: http://www.flacso.org.ar/rrii/web/wp-content/uploads/2010/09/ Kiessling-Crisis-ambiental-global-COP-15.pdf

LAFFERRIERE, R., El mecanismo de Desarrollo Limpio del Protocolo de Kyoto. Ed. CO2- EcoConsulting, Argentina, 2008.

MEJÍA, C., "Mecanismo de Desarrollo Limpio: Una debilidad convertida en oportunidad", en Pensamiento y gestión: Revista de la División de Ciencias Administrativas de la Universidad del Norte, núm. 18, Colombia, 2005.

SINDICO, F., "The Copenhagen Accord and the future of the international climate change regime", en Revista Catalana de Dret Ambiental, vol. I - núm. 1, 2010. Recuperado el 23 de agosto de 2010, de: http://www.rcda.cat. 
URRUTIA, O., "El régimen jurídico internacional del cambio climático después del "Acuerdo de Copenhague", en Revista de Derecho de la Pontificia Universidad Católica de Valparaíso, núm. 34, 2010. Recuperado el 27 de septiembre de 2010, de: http://www.scielo.cl/scielo.php?pid=S0718-68512010000100019\&script=sci_abstract.

WERKSMAN, J., "The Clean Development Mechanisms: Unwrapping the "Kyoto Surprise", en Review of European Community and International Environmental Law, vol. 7, issue 2, 1998.

WOERDMAN, E., "Hot Air Trading under the Kyoto Protocol: An Environmental Problem or Not?”, en European Environmental Law Review, vol. 14, 2005. Recuperado el 10 de septiembre de 2010, de: http://rechten.eldoc.ub.rug.nl.

\section{Referencias documentales}

AMIGOS DE LA TIERRA BRASIL. REDD y el futuro de los bosques, Porto Alegre, Brasil, 2007. Recuperado el 5 de octubre de 2010, de: http://www.natbrasil.org.br/ cartilhareddweb.pdf.

ASAMBLEA GENERAL DE LAS NACIONES UNIDAS, Resolución A/RES/63/278 de 1 de mayo de 2009, relativa al "Día Internacional de la Madre Tierra". Recuperado el 27 de septiembre de 2010, de: http:/www.un.org/es/comun/docs/?symbol=A/RES/ $63 / 278$.

ASOCIACIÓN INTERNACIONAL DE GESTORES DE EMERGENCIA (IAEMEspaña), Incendios Forestales en Rusia, informe actualizado el 8 de septiembre de 2010, Madrid, España, 2010.

BBC MUNDO (22 de agosto de 2010), "Corea del Norte y China afectadas por inundaciones". Recuperado el 20 de septiembre de 2010, de: http://www.bbc.co.uk/ mundo/internacional/2010/08/100822_inundaciones_china_corea_norte_aw.shtml.

- (22 de abril 2010), "Un clima polarizado: Cochabamba y Copenhague". Recuperado el 29 de septiembre de 2010, de: http://www.bbc.co.uk/mundo/ $\begin{array}{lllllllllllllllllll}\mathrm{c} & \mathrm{i} & \mathrm{e} & \mathrm{n} & \mathrm{c} & \mathrm{i} & \mathrm{a} & \ldots & \mathrm{t} & \mathrm{e} & \mathrm{c} & \mathrm{n} & \mathrm{o} & 1 & \mathrm{l} & \mathrm{g} & \mathrm{i} & \mathrm{a} & /\end{array}$ 2010/04/100422_2144_internacional_bolivia_cumbre_copenhague_fp.shtml. 
CAN INTERNATIONAL, "Fossil of the Day", Bonn, Alemania, 2 - 2010. Recuperado el 28 de septiembre de 2010, de: http://www.climatenetwork.org/event/bonn-2-2010.

CENTRO HUMBOLDT Para la promoción del desarrollo territorial y la gestión ambiental, "Finaliza Bonn II con un Copenhagen Accord Plus". Recuperado el 3 de octubre de 2010, de: http://www.humboldt.org.ni/blog/2010/06/14/finaliza-bonn-ii-conun-copenhaguen-accord-plus.

\section{CONFERENCIA MUNDIAL DE LOS PUEBLOS SOBRE EL CAMBIO CLIMÁTICO}

Y LOS DERECHOS DE LA MADRE TIERRA, "Convocatoria de la Conferencia Mundial de los Pueblos sobre el Cambio Climático y los Derechos de la Madre Tierra", de 5 de enero de 2010. Recuperado el 23 de septiembre de 2010, de: http://cmpcc.org/ 2010/01/07/convocatoria/\#more-1.

— "Conclusiones finales de los Grupos de Trabajo de la CMPCC", de 28 de abril de 2010. Recuperado el 23 de septiembre de 2010, de: http://cmpcc.org/category/ grupos-de-trabajo/.

- "Comunicado del Estado Plurinacional de Bolivia: las propuestas de Cochabamba van a Cancún", de 10 de octubre de 2010. Recuperado el 14 de octubre de 2010, de: http://cmpcc.org/2010/10/10/comunicado-del-estado-plurinacional-de-bolivia/ \#more-2397.

CONVENIO MARCO DE NACIONES UNIDAS SOBRE EL CAMBIO CLIMÁTICO, Decisión 2/CP.15 Acuerdo de Copenhague, del Doc. FCCC/CP/2009/11/Add.1 de 30 de marzo de 2010. Recuperado el 20 de septiembre de 2010, de: http://unfccc.int/resource/ $\operatorname{docs} / 2009 /$ cop15/spa/11a01s.pdf\#page $=4$.

— Doc. FCCC/CP/2007/6/Add.1, Decisión 1/CP.13 de 14 de marzo de 2008, pp. 3-8. Recuperado el 20 de septiembre de 2010, de: http://unfccc.int/resource/docs/2007/ cop13/spa/06a01s.pdf\#page $=3$.

— "Discurso público de Yvo de Boer, secretario ejecutivo", Bruselas, 14 de abril de 2010. Recuperado el 27 de septiembre de 2010, de: http://unfccc.int/files/ portal_espanol/press/statements/application/pdf/100414_speech_brussels_spa.pdf. 
El Mundo (23 de julio de 2010), "EEUU no tendrá ley contra el cambio climático". Recuperado el 1 de octubre de 2010, de: http://www.elmundo.es/america/2010/07/23/ estados_unidos/1279920449.html.

- (19 de diciembre de 2009), "Un avance significativo, pero insuficiente". Recuperado el 12 de septiembre de 2010, de: http://www.elmundo.es/elmundo/ 2009/12/18/ciencia/1261172856.html.

EUROPA PRESS (6 de agosto de 2010), "Finaliza la reunión de la ONU sobre el cambio climático, con más retrocesos que avances". Recuperado el 21 de septiembre de 2010, de: http://www.europapress.es/internacional/noticia-finaliza-reunion-onu-cambioclimatico-mas-retrocesos-avances-20100806230411.html.

INSTITUTO INTERNACIONAL PARA EL DESARROLLO SOSTENIBLE (IIDS), "Conversaciones de Bonn sobre el clima", en Boletín de Negociaciones de la Tierra, vol. 12, núm. 461, 2010. Recuperado el 25 de septiembre de 2010, de: http:// www.iisd.ca/climate/sb32/compilations.pdf.

— "Sumary of the Bonn Climate Change Talks: 9-11 April 2010", en Earth Negotiations Bulletin, vol. 12, núm. 460, 2010. Recuperado el 25 de septiembre de 2010, de: http://www.iisd.ca/download/pdf/enb12460e.pdf (25/10/2010).

— "Síntesis de las conversaciones de Bonn sobre el cambio climático: 2 al 6 de agosto de 2010”, en Boletín de Negociaciones de la Tierra, vol. 12, núm. 478, 2010. Recuperado el 1 de octubre de 2010, de: http://www.iisd.ca/download/pdf/ enb12478s.pdf.

_ “Tianjin Climate Change Talks: 4.9 October 2010”, en Earth Negotiations Bulletin, vol. 12, núm 479, 2010. Recuperado el 12 de octubre de 2010, de: http:// www.iisd.ca/climate/ccwg12/compilatione.pdf.

INTERGOVERNMENTAL PANEL ON CLIMATE CHANGE (IPCC), Third Assessment Report: Climate Change 2001 (TAR). Recuperado el 9 de septiembre de 2010, de: http://www.grida.no/climate/ipcc_tar/vol4/spanish/pdf/wg3sum.pdf.

INTERNATIONAL CENTRE FOR TRADE AND SUSTAINABLE DEVELOPMENT, "Year-End Climate Goal in Question as Bonn Meetings Begin", en Bridges Weekly 
Trade News Digest, vol. 14, núm. 20, 2010. Recuperado el 20 de septiembre de 2010, de: http://ictsd.org/i/news/puentesquincenal/77648.

La Razón (12 de junio de 2010), "Protocolo de Kyoto sería excluido en Cancún". Recuperado el 22 de septiembre de 2010, de: http://www.la-razon.com/version.php? ArticleId=3356\&EditionId=104.

La Vanguardia (7 de octubre de 2010) "China condiciona la reducción de sus emisiones al dinero y la tecnología que reciba”. Recuperado el 12 de octubre de 2010, de: http:// www.lavanguardia.es/ciudadanos/noticias/20101007/54020261184/china-condiciona-lareduccion-de-sus-emisiones-al-dinero-y-la-tecnologia-que-reciba.html.

Los tiempos (4 de julio 2010), "Las negociaciones de Bonn y el cambio climático". Recuperado el 27 de septiembre de 2010, de: http://www.lostiempos.com/oh/actualidad/ actualidad/20100704/las-negociaciones-de-bonn-y-el-cambioclimatico_78283_147654.html.

NOAA, "Second Warmest July and Warmest Year-to-Date Global Temperature on Record", de 13 de agosto de 2010. Recuperado el 5 de octubre de 2010, de: http:// www.noaanews.noaa.gov/stories2010/20100813_globalstats.html.

THE WORLD BANK, Prototype carbon fund - Annual Report, Washington, DC, 2004. Recuperado el 26 de septiembre de 2010, de: http://wbcarbonfinance.org/docs/ 2004PCFAR.pdf.

\section{Referencias normativas}

Convención de Viena sobre el Derecho de los Tratados, de 23 de mayo de 1969 (BOE núm. 142, de 13 de junio de 1980).

Protocolo de Kioto del Convenio Marco de las Naciones Unidas sobre el Cambio Climático (BOE núm. 33, de 8 de febrero de 2005). 